\title{
Charged lepton contributions to the solar neutrino mixing and $\boldsymbol{\theta}_{13}$
}

\author{
Andrea Romanino \\ CERN, Department of Physics, Theory Division, CH-1211 Geneva 23, Switzerland
}

(Received 3 March 2004; published 27 July 2004)

\begin{abstract}
A charged lepton contribution to the solar neutrino mixing induces a contribution to $\theta_{13}$, barring cancellations or correlations, which is independent of the model building options in the neutrino sector. We illustrate two robust arguments for that contribution to be within the expected sensitivity of high intensity neutrino beam experiments. We find that the case in which the neutrino sector gives rise to a maximal solar angle (the natural situation if the hierarchy is inverse) leads to a $\theta_{13}$ close to or exceeding the experimental bound depending on the precise values of $\theta_{12}, \theta_{23}$, an unknown phase and possible additional contributions. We finally discuss the possibility that the solar angle originates predominantly in the charged lepton sector. We find that the construction of a model of this sort is more complicated. We comment on a recent example of natural model of this type.
\end{abstract}

DOI: 10.1103/PhysRevD.70.013003

PACS number(s): $14.60 . \mathrm{Pq}$

\section{INTRODUCTION}

The steady, remarkable progress we have witnessed in recent years in experimental neutrino physics has enabled a significant advance in our understanding of the lepton sector. The case for three neutrino oscillations is now compelling (although a full oscillation pattern still has to be observed) and the peculiar neutrino mass and mixing pattern observed represents a nontrivial handle on their origin. It is then natural to wonder what the understanding we gained implies for the value of the observables still to be measured, in particular $\theta_{13}$. A major part of the rich neutrino experimental program available and partially under way will in fact focus on measuring that mixing angle, which has also important implications for leptonic $C P$ violation and astrophysics. While many models do provide predictions for $\theta_{13}$, the number of possibilities is high enough to make almost any value of $\theta_{13}$, from zero to the present bound, compatible with some model. In this paper, we will therefore try to overcome the bulk of the model dependence in those predictions by focusing on general mechanisms leading to calculable contributions to $\theta_{13}$.

While both the neutral and charged lepton sectors contribute to lepton mixing, most of the uncertainties in model building come from the neutrino sector. This is because the light neutrino mass matrix is still less constrained than the charged lepton one and, more important, because the origin of its smallness introduces additional degrees of freedom. For example, in the case of the type I see-saw mechanism, the light neutrino matrix is determined by two independent mass matrices. ${ }^{1}$ We will therefore concentrate on contributions to $\theta_{13}$ that are independent of the model building in the neutrino sector and rely instead on properties of the charged

\footnotetext{
${ }^{1}$ One way of getting oriented in the jungle of model building possibilities in the neutrino sector is by considering minimal models [1]. See also the general arguments in [2-5]. In [6] the assumption is made that the neutrino contribution to the mixing matrix is bimaximal. In some cases the model building in the neutrino and charged lepton sector can be closely related [7].
}

lepton sector. ${ }^{2}$ In particular, we identify a contribution to $\theta_{13}$ induced by the charged lepton contribution to the solar mixing angle, which arises in the absence of correlations in the charged lepton mass matrix. We discuss two motivated expectations for the size of the latter contribution, both leading to values of $\theta_{13}$ likely to be within the reach of high intensity conventional neutrino beam experiments. In particular, we consider the case in which the neutrino sector gives rise to a maximal solar angle. This is typically the case in models with inverse hierarchical neutrinos [11]. In this case, the charged lepton sector must account for the observed deviation of $\theta_{12}$ from $45^{\circ}$, which in turn leads to a contribution to $\theta_{13}$ close to the present experimental bound $[11,12]$. We provide a simple analytical expression for the latter involving one physical phase and we plot the distribution of the corresponding numerical expectation taking into account the uncertainty on the mixing parameters and possible additional contributions [13]. Indeed, since additional contributions to $\theta_{13}$ may be present, accidentally canceling the piece controlled by the charged lepton sector, the expectations we find should be considered as lower limits on $\theta_{13}$, uncertain by a factor of order one. A condition for a nonaccidental cancellation is discussed in [14].

We also discuss the possibility that the charged lepton contribution to the solar mixing angle is large and accounts for most of it. We first consider the case in which the entries in the charged lepton mass matrix are uncorrelated and show that (i) the induced contribution to $\theta_{13}$ is well beyond the experimental bound (the " $\theta_{13}$ tuning problem") and that (ii) in SU(5) grand unified models, the electron mass (or the up quark mass) gets a contribution way larger than the observed value (the " $m_{e}$ tuning problem," numerically more relevant). This makes the construction of a natural model of this sort considerably more complicated [13]. We then discuss the case in which correlations are present in the charged lepton mass matrix. In particular, we consider the possibility, re-

\footnotetext{
${ }^{2}$ This contribution arises in a number of explicit models, in some cases providing a precise prediction for $\theta_{13}$, see e.g. $[8,3,9,10,6]$. We focus here on the general features of the effect.
} 
cently pointed out in [14], that such correlations arise from a non-accidental physical mechanism. A condition on the charged lepton mass matrix sufficient to naturally solve the $\theta_{13}$ tuning problem has been identified in [14]. We show that an additional condition is necessary to ensure that the $m_{e}$ tuning problem is also solved in SU(5) models. The mechanism illustrated in [14] may easily account for the latter condition as well.

\section{CHARGED LEPTON ROTATIONS AND $\theta_{13}$}

The neutrino mixing matrix $U$ is the combination of two unitary matrices, $U_{e}$ and $U_{\nu}$, entering the diagonalization of the charged lepton and neutrino mass matrices $m_{E}$ and $m_{\nu}{ }^{3}$

$$
\begin{gathered}
U=U_{e} U_{\nu}^{\dagger}, \quad \text { where } m_{E}=U_{e_{c}}^{T} m_{E}^{\mathrm{diag}} U_{e}, \\
m_{\nu}=U_{\nu}^{T} m_{\nu}^{\mathrm{diag}} U_{\nu} .
\end{gathered}
$$

The decomposition of $U$ into $U_{e}$ and $U_{\nu}$ is of course not physical in the (minimally extended) standard model (SM). ${ }^{4}$ On the other hand, any attempt at investigating the origin of the fermion flavor structure involves physics beyond the SM that identifies a privileged basis in flavor space. This is the basis in which the entries of the fermion mass matrices are most simply related to the physics originating them and in which possible correlations among the entries in $m_{E}, m_{\nu}$ have to be considered accidental or related to symmetries or other physical mechanisms of the underlying theory.

We can then wonder if lepton mixing, and in particular the two large mixing angles observed in atmospheric, solar and terrestrial neutrino experiments, originate from the neutrino or the charged lepton sector. It is well known that the atmospheric angle $\theta_{23}$ can equally well originate from the neutrino [15] or the charged lepton [16] sector. The solar angle can in principle also come from both sectors. However, as we will see later on, cancellations or correlations in the charged lepton mass matrix are required in the case in which the origin of the solar angle is in the lepton sector. For the time being, we consider the case in which the entries of $m_{E}$ are not correlated and show that in general a left-handed 12-rotation in the charged lepton sector $\theta_{12}^{e}$ induces a contribution to $\theta_{13}$. This derivation is closely related to that in [3].

Barring correlations in the charged lepton mass matrix $m_{E}{ }^{5}$, the hierarchy of charged lepton masses translates in a hierarchical structure of $m_{E}$ :

\footnotetext{
${ }^{3}$ We use a convention in which the left-handed fields lie on the right-hand side of $m_{E}$.

${ }^{4} \mathrm{An} S U(2)_{L}$-invariant transformation in flavor space can equally well rotate the physical mixing in the neutrino and in the charged lepton sector.

${ }^{5}$ Democratic models are an example in which such correlations are not accidental.
}

$$
\left|\begin{array}{lll}
m_{11} & m_{12} & m_{13} \\
m_{21} & m_{22} & m_{23} \\
m_{31} & m_{32} & m_{33}
\end{array}\right|^{E} \ll\left|\begin{array}{cc}
m_{22} & m_{23} \\
m_{32} & m_{33}
\end{array}\right|^{E} m_{\tau} \ll\left|m_{33}\right|^{E} m_{\tau}^{2} .
$$

Note that the condition (2) is compatible with asymmetrical matrices and in particular with the atmospheric angle originating from $m_{E}$.

The charged lepton mass matrix can be approximately diagonalized by the subsequent diagonalization of his $2 \times 2$ blocks. As a consequence of Eq. (2), the diagonalization of the heaviest 23 block should be performed first, so that

$$
U_{e}=U_{12}^{e} U_{13}^{e} U_{23}^{e}
$$

where $U_{i j}^{e}$ is a complex rotation in the $i j$ block. The ordering (3) ensures that the mixing parameters are directly related to the entries of $m_{E}$ and are therefore independent, barring correlations already present in $m_{E}$ or induced by the first steps of diagonalization. For our purposes, it is sufficient to consider the two cases $U_{12}^{e} \neq \mathbf{1}, U_{13}^{e}=\mathbf{1}$ and $U_{13}^{e} \neq \mathbf{1}, U_{12}^{e}=\mathbf{1}$. Since the two possibilities are actually equivalent (up to a relabelling of the second two rows of $m_{E}$ ) in the $\theta_{23}=\pi / 4$ limit, in this paper we will consider the $U_{12}^{e} \neq \mathbf{1}, U_{13}^{e}=\mathbf{1}$ case only.

When combining $U_{e}$ and $U_{\nu}$ in the physical neutrino mixing matrix, the $U_{12}^{e}$ rotation ends up on the left-hand side of $U$ :

$$
U=U_{12}^{e} \hat{U}
$$

where $\hat{U}=U_{23}^{e} U_{\nu}^{\dagger}$ and the standard parametrization can be used for $U_{\nu}$. Note that, wherever it comes from, the atmospheric angle resides in $\hat{U}$. Now, in order to read the value of the solar angle $\theta_{12}$ from Eq. (4) we have to write $U$ in the parametrization that defines $\theta_{12}$, in which the 12 rotation lies on the right-hand side: $U=U_{23} U_{13} U_{12}$. This means that $U_{12}^{e}$ has to be commuted with $\hat{U}$ and in particular with the large 23 rotation, thus inducing a contribution $\widetilde{\theta}_{13}$ to $\theta_{13}$ which is easily found to be given by

$$
\sin \widetilde{\theta}_{13}=\sin \theta_{12}^{e} \frac{\tan \theta_{23}}{\sqrt{\cos ^{2} \theta_{12}^{e}+\tan ^{2} \theta_{23}}} \simeq \sin \theta_{12}^{e} \sin \theta_{23}
$$

where $\theta_{12}^{e}$ is the angle associated to $U_{12}^{e}$ and the approximated expression holds for small $\theta_{12}^{e}$. Eq. (5) generalizes results in $[3,17,10]$. By construction, under the present assumptions the contribution in Eq. (5) is independent of possible additional contributions to $\theta_{13}$. A cancellation among them would be accidental.

The contribution to $\theta_{13}$ we obtain this way does not depend on the many unknowns associated with the model building in the neutrino sector. In particular, it is independent of the form of the light neutrino mass matrix; of the mechanism accounting for its smallness; of the form of the Majorana and Dirac mass matrices in see-saw models. It is also independent of the origin of the atmospheric mixing angle (neutrino or charged lepton sector). 


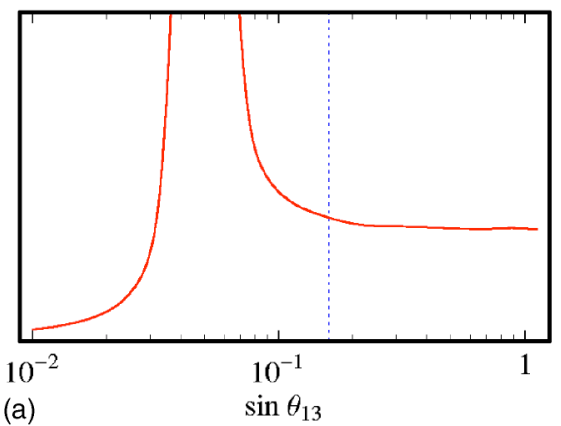

FIG. 1. Distribution of $\theta_{13}$ obtained by adding a random contribution to $\sin \widetilde{\theta}_{13}$ $=\sqrt{m_{e} / m_{\mu}} \sin \theta_{23}$ (a) or $\sin \widetilde{\theta}_{13}$ $=\tan \theta_{23} / 2 \cos 2 \theta_{12} / \cos \phi \quad$ (b). The vertical dotted line represents the present limit. The case $\cos \phi$ $=1$ (solid) and $\cos \phi=0.5$ (dashed) are both shown in (b). See comments in the text.
On the other hand, $\widetilde{\theta}_{13}$ does depend on properties of the charged fermion sector, such as the size of the $\theta_{12}^{e}$ rotation and the independence of the entries of $m_{E}$. In the next section we illustrate two arguments on the expected size of $\theta_{12}^{e}$.

\section{TWO ARGUMENTS ON THE SIZE OF $\theta_{12}^{E}$}

We first consider a well known, robust ansatz on the structure of the light $2 \times 2$ block of the charged fermion mass matrices. The ansatz is characterized by a negligible 11 element and by the approximate equality of the absolute values of the 12 and 21 elements [18,9]. Such a pattern can be accounted for by an elegant non-Abelian symmetry [19]. More important, it leads to the successful and precise (at the $5 \%$ level) relation $\left|V_{u s}\right|=\sqrt{m_{d} / m_{s}}$. Furthermore, in a SU(5) grand unified model, the relations $m_{12(21)}^{E}=m_{21(12)}^{D}$, together with the Georgi-Jarlskog factor 3 in $m_{22}^{E}=3 m_{22}^{D}$ (necessary to account for the muon mass), lead to a second successful prediction: $m_{e} / m_{\mu}=\left(m_{d} / m_{s}\right) / 9$. A prediction for $\theta_{12}^{e}$ also follows, which can certainly be considered well motivated, in the light of the above. The prediction is $\theta_{12}^{e}$ $=\left(m_{e} / m_{\mu}\right)^{1 / 2} \simeq 0.07[8,9]$, leading to

$$
\sin \widetilde{\theta}_{13} \simeq \sqrt{m_{e} / m_{\mu}} \sin \theta_{23} \simeq 0.05,
$$

3-4 times below the present experimental value and therefore within the reach of future high intensity neutrino beam experiments.

In the presence of additional contributions, Eq. (6) should be interpreted as a lower bound on $\theta_{13}$ that can be evaded if a cancellation occurs. In order to estimate how small $\theta_{13}$ can be made by such a cancellation, we add to $\sin \widetilde{\theta}_{13}$ a random contribution larger than $10^{-4}$, with flat logarithmic distribution and arbitrary phase, in such a way that the additional contribution alone would have a flat distribution in the logarithmic scale of Fig. 1. We then obtain a probability distribution for $\theta_{13}$, which is shown in Fig. 1(a). The peak at $\theta_{13} \simeq \widetilde{\theta}_{13}$ is due to the small values of the additional contribution (which all give $\theta_{13} \simeq \widetilde{\theta}_{13}$ ) and is therefore not particularly meaningful. More meaningful is the range of $\theta_{13}$ in which the probability distribution is not too much suppressed with respect to the plateau on the right of the peak. We find that the suppression factor is larger than 5 for $\theta_{13}<0.02$. In other words, a cancellation leading to $\theta_{13}<0.02$ is unlikely. Figure 1(a) is a (mild) generalization of Fig. 1(b) in [9], where the additional contribution comes from a specific structure of the neutrino mass matrix. See also $[3,10]$.

The case in which the neutrino mass spectrum is of the inverted type provides a different, purely phenomenological constraint on $\theta_{12}^{e}$. Strictly speaking, the argument follows from assuming that the matrix $\hat{U}$ in Eq. (4) is in the form $\hat{U}=U_{23}(\theta) U_{12}\left(\theta_{12}=\pi / 4\right)$. This is the typical situation in models leading to the inverted neutrino mass pattern. In such models, in fact, the deviation of the neutrino contribution to the solar angle from $\pi / 4$ is related to $\Delta m_{21}^{2} / \Delta m_{32}^{2}$ and turns out to be small compared to the observed deviation [11], barring tunings. ${ }^{6}$ Depending on the model, the atmospheric angle can come from the neutral or the charged lepton sector (or both), but in both cases $\hat{U}$ has the above form, up to a possible further 13 rotation, which we neglect. A sizable $\theta_{12}^{e}$ is required in this case to account for the observed value of $\theta_{12}$, significantly different from $\pi / 4[11,12]$. The relation between the deviation of $\theta_{12}$ from $\pi / 4$ and the required $\theta_{12}^{e}$ involves a physical phase $\phi[1,13]$ :

$$
\begin{aligned}
& \sin \theta_{13}=\sin \theta \sin \theta_{12}^{e}, \\
& \tan \theta_{23}=\cos \theta_{12}^{e} \tan \theta, \\
& \tan \theta_{12}=\left|\frac{1-\cos \theta \tan \theta_{12}^{e} e^{i \phi}}{1+\cos \theta \tan \theta_{12}^{e} e^{i \phi}}\right| .
\end{aligned}
$$

Solving for $\theta_{12}^{e}$, we obtain the following equation for $\sin \theta_{12}^{e} / \sqrt{\cos ^{2} \theta_{12}^{e}+\tan ^{2} \theta_{23}} \equiv x=\sin \theta_{13} / \tan \theta_{23}$ :

$$
\frac{2 x}{1+x^{2}}=\frac{\cos 2 \theta_{12}}{\cos \phi}
$$

or, neglecting terms quadratic in $x$,

$$
\sin \theta_{12}^{e}=\frac{\cos 2 \theta_{12}}{2 \cos \theta_{23} \cos \phi} .
$$

\footnotetext{
${ }^{6}$ If the atmospheric angle comes from the charged lepton sector, the 11 and 22 elements of the neutrino mass matrix should to be tuned to be equal up to a phase. This could be obtained in a nonaccidental way by using a non-Abelian symmetry.
} 
At the same order, $\theta_{13}$ is given by

$$
\sin \theta_{13}=\frac{\tan \theta_{23}}{2} \frac{\cos 2 \theta_{12}}{\cos \phi} .
$$

In explicit models, the phase $\phi$ can be related to the leptonic $C P$-violating phase in the standard parametrization [1]. Numerically, the prediction for $\theta_{13}$ is very close to or beyond the experimental bound, depending on $\cos \phi$ and on the values used for $\theta_{12}, \theta_{23}$. In Fig. 1(b) we show the distribution for $\sin \theta_{13}$ we obtain by using the present distributions for $\theta_{23}$ and $\theta_{12}$ and by adding a random contribution as before. The solid line corresponds to $\cos \phi=1$, whereas the dashed line corresponds to $\cos \phi=0.5$. In the case $\cos \phi=1$, the probability is suppressed by a factor of more than 5 (compared with the plateau on the right of the peak) when $\theta_{13}<0.07$. When $\cos \phi<0.5$, this happens in the whole allowed range.

Note that Eq. (9) is not compatible with the powerful ansatz described at the beginning of this section, since it requires a larger $\theta_{12}^{e}\left(\theta_{12}^{e} \sim 0.25-0.30\right)$. In order to avoid a large contribution to the electron mass, this in turn requires a significant asymmetry in the 12 block of $m_{E}$.

\section{IV. $\theta_{12}$ FROM THE CHARGED LEPTON SECTOR (NO CORRELATIONS IN $m_{E}$ )}

Let us now consider the possibility that the solar angle originates predominantly in the charged lepton sector. We first consider again the case in which the entries in $m_{E}$ are independent. Then $\theta_{12}$ must originate from the $U_{12}^{e}$ factor in Eq. (4). Since the required size of the $\theta_{12}^{e}$ rotation is now quite large,

$$
\sin \theta_{12}^{e}=\frac{\sin \theta_{12}}{\cos \theta_{23}} \simeq 0.8,
$$

we can expect the induced contribution to $\theta_{13}$ to be large. Indeed, in the limit in which the matrix $\hat{U}$ in Eq. (4) consists of a pure 23 rotation, we have

$$
\sin \widetilde{\theta}_{13}=\tan \theta_{23} \tan \theta_{12} \simeq 0.6-0.7,
$$

4-5 times above the experimental limit. Therefore, the matrix $\hat{U}$ should contain an additional 13 or 12 rotation factor cancelling most of $\widetilde{\theta}_{13}$. We will refer to this problem as the " $\theta_{13}$ tuning problem." In this scenario, one of the few hints on the origin of lepton mixing (the smallness of $\theta_{13}$ ) would be an accident. On the other hand, the degree of cancellation needed to bring $\theta_{13}$ below the experimental limit is mild. A potentially more serious tuning problem comes from the $m_{e} \ll m_{\mu}$ hierarchy, at least in SU(5) models, as we now see.

Let us call $\theta_{12}^{e_{c}}$ the right-handed rotation involved in the diagonalization of the light $2 \times 2$ block of the charged lepton matrix (after diagonalization of the 23 block). The $e / \mu$ mass ratio is then given by

$$
\frac{m_{e}}{m_{\mu}} \simeq\left|\frac{m_{11}^{E}}{\hat{m}_{22}^{E}}-\tan \theta_{12}^{e} \tan \theta_{12}^{e_{c}}\right|,
$$

where $\hat{m}_{22}^{E}$ is the 22 entry of $m_{E}$ after diagonalization of the 23 block. As a consequence, we expect $m_{e} / m_{\mu}$ to be of the order of $\tan \theta_{12}^{e} \tan \theta_{12}^{e} \sim \tan \theta_{12}^{e_{c}}$ or larger, barring cancellations. On the other hand, $\theta_{12}^{e_{c}}$ is related to the left-handed 12 rotation in the down quark sector $\theta_{12}^{d}$ (analogous of $\theta_{12}^{e}$ ) by GUT relations: $\theta_{12}^{d}=C \theta_{12}^{e}$, where $C$ comes from possible SU(5) Clebsch-Gordan factors. We also know that $\theta_{12}^{d}$ contributes to the CKM element $V_{u s}$. Indeed, if the up quark mass is to be naturally small, the up quark contribution to $V_{u s}$ must be subdominant. That is because the up quark matrix is symmetrical in $\mathrm{SU}(5)$ and $\theta_{12}^{u} \sim\left|V_{u s}\right|$ would give a large contribution to $m_{u} / m_{c}$ through a relation similar to Eq. (13). We then have $\theta_{12}^{d} \simeq\left|V_{u s}\right|$ and a contribution to $m_{e} / m_{\mu}$ of order $\left|V_{u s}\right| / C$. In minimal SU(5), this would be about 50 times larger than the measured value. Even in the presence of a (plausible) Clebsch-Gordan factor, the necessary tuning is still larger than that involved in the $\theta_{13}$ tuning problem. We refer to this problem as the " $m_{e}$ tuning problem."

In summary, in absence of correlations in $m_{E}$, generating the solar mixing angle from the charged lepton sector requires cancellations in the determination of $\theta_{13}$ and, in SU(5) models, in the determination of the electron (or up quark) mass.

\section{V. $\theta_{12}$ FROM CORRELATIONS IN $m_{E}$}

Let us now consider the case in which the entries of the charged lepton mass matrix are correlated. It has recently been shown [14] that such correlations can be naturally induced in a Froggat-Nielsen context by the dominance of a heavy vector-like lepton exchange (analogous to the single right-handed neutrino dominance scenario in the neutrino sector [15]). More precisely, the approximate vanishing of the determinant of the $x_{i j}$ coefficients in

$$
m_{E} \propto\left(\begin{array}{ccc}
a \epsilon^{\prime} & b \epsilon^{\prime} & \mathcal{O}\left(\epsilon^{\prime}\right) \\
x_{21} \epsilon & x_{22} \epsilon & \mathcal{O}(\epsilon) \\
x_{31} & x_{32} & 1
\end{array}\right)
$$

( $\epsilon^{\prime} \ll \epsilon \ll 1 \sim a, b, x_{i j}$ ) has been identified as a condition for the large $\theta_{12}$ (and $\theta_{23}$ ) to originate in a natural way from $m_{E}$. The correlations in $m_{E}$ translate in fact in a relation between the 12, 13, 23 rotations in Eq. (3) cancelling the physical $\theta_{13}$ angle. This can easily be seen by observing that, unlike the case in which correlations are absent, the proper way to diagonalize $m_{E}$ is by performing the left-handed 12 rotation first, followed by the 23 rotation.

The form of $m_{E}$ in Eq. (14) is not unique. For example, the pattern

$$
\left(\begin{array}{ccc}
\epsilon^{\prime} & 0 & x_{13} \epsilon \\
0 & \epsilon^{\prime} & x_{23} \epsilon \\
x_{31} & x_{32} & 1
\end{array}\right)
$$

with $\left|x_{13} x_{32}-x_{31} x_{23}\right| \ll 1$, also gives a large solar angle in a natural way. However, it is not compatible with SU(5) (see below) and it is harder to obtain from the model building 
point of view (see however [7]). Another possibility is that correlations are not present in the initial form of $m_{E}$ but they are induced by the first steps of the diagonalization in Eq. (3). In the following we will concentrate on the possibility in Eq. (14).

While the $\left|x_{21} x_{32}-x_{31} x_{22}\right| \ll 1$ condition on the coefficients in Eq. (14) does solve the $\theta_{13}$ problem discussed in the previous section, the additional condition

$$
\left|a x_{22}-b x_{21}\right| \ll 1
$$

must be imposed in order to ensure that the electron mass and the up quark mass are naturally small in $\mathrm{SU}(5)$ grand unified models (namely to ensure that the $m_{e}$ problem illustrated above is also solved). The only possibility to escape the condition (16) is boosting the 13 entry in Eq. (14), namely imposing the alternative condition $\left|m_{11}^{E}\right|,\left|m_{12}^{E}\right|$ $\ll\left|m_{13}^{E}\right|$.

The argument goes as follows. In SU(5) the light block of the up quark mass matrix is symmetric. As a consequence, the up quark contribution to $\left|V_{u s}\right|$ must be subdominant, as discussed in the previous section, and we can identify $\left|V_{u s}\right|$ with its down quark contribution. We then obtain, e.g. in minimal SU(5),

$$
\frac{m_{e}}{m_{\mu}} \cos \theta_{23} \sim\left|V_{u s} \frac{a x_{22}-b x_{21}}{a x_{21}+b x_{22}}\right| .
$$

The condition (16) follows from $\left|V_{u s}\right| \gg m_{e} / m_{\mu} \cos \theta_{23}$. We have used $\theta_{12}^{e_{c}} \simeq\left|V_{u s}\right|$ and we have neglected the contribution of the 13 entry in Eq. (14) to $\theta_{12}^{e} c$ that arises due to the 23 rotation. If that entry is larger than $\mathcal{O}\left(\epsilon^{\prime}\right)$, its contribution to $\theta_{12}^{e}$ and $V_{u s}$ dominates and the connection with $m_{e} / m_{\mu}$ is lost, thus leading to the alternative possibility $\left|m_{11}^{E}\right|,\left|m_{12}^{E}\right|$ $\ll\left|m_{13}^{E}\right|$.

The above can be rephrased by using the example in Sec. I of [14]. Assume that the lepton mixing matrix is in the form $U=U_{23}\left(\theta_{23}=\pi / 4\right) U_{12}(\theta)$. Then, using the notation of Eq. (4) in [14],

$$
m_{E}=V_{e}\left(\begin{array}{ccc}
c m_{e} & s m_{e} & 0 \\
s / \sqrt{2} m_{\mu} & -c / \sqrt{2} m_{\mu} & m_{\mu} / \sqrt{2} \\
-s / \sqrt{2} m_{\tau} & c / \sqrt{2} m_{\tau} & m_{\tau} / \sqrt{2}
\end{array}\right) .
$$

Given the relation between $\left(V_{e}\right)_{12}$ and $V_{u s}$, taking $V_{e} \sim 1$ is not a good approximation in SU(5). For instance, in minimal SU(5) we have

$$
m_{E} \simeq\left(\begin{array}{ccc}
V_{u s} s / \sqrt{2} m_{\mu} & -V_{u s} c / \sqrt{2} m_{\mu} & V_{u s} m_{\mu} / \sqrt{2} \\
s / \sqrt{2} m_{\mu} & -c / \sqrt{2} m_{\mu} & m_{\mu} / \sqrt{2} \\
-s / \sqrt{2} m_{\tau} & c / \sqrt{2} m_{\tau} & m_{\tau} / \sqrt{2}
\end{array}\right) .
$$

Additional contributions to the first and second rows proportional to $V_{u b}$ and $V_{c b}$ might also be relevant, but they have been omitted in Eq. (19).

From the model building point of view, the additional condition (16) does not represent an additional challenge. The same mechanism accounting for the correlation in the lower left block of $m_{E}$ may well account also for the correlation in the upper left block. This is illustrated by the explicit SU(5) model in Appendix A of [14]. In the light of Eq. (19), we remark however that one might expect the first row of $m_{E}$ to be suppressed by only a factor $\left|V_{u s}\right|$ compared to the second row.

\section{SUMMARY}

We have shown that a charged lepton contribution to the solar neutrino mixing induces a contribution to $\theta_{13}$, barring cancellations or correlations, which is independent of the model building options in the neutrino sector. We have illustrated two robust arguments for that contribution to be within the expected sensitivity of high intensity conventional neutrino beam experiments. The corresponding expectations for $\theta_{13}$ are shown in Fig. 1. In particular, in the case in which the neutrino sector gives rise to a maximal solar angle (the natural situation if the neutrino spectrum is inverted, barring nonaccidental tunings) we have given a simple analytical expression for the induced contribution to $\theta_{13}$ taking into account the dependence on a physical phase. The numerical value of $\theta_{13}$ turns out to be very close or exceeding the experimental bound, depending on the precise value of the solar and atmospheric mixing angles, the value of the phase, and the presence of additional contributions. We have also discussed the possibility that the solar angle originates predominantly in the charged lepton sector. In the case in which no correlations are forced in the charged lepton mass matrix, we have shown that this possibility faces two fine-tuning problems, one in the determination of $\theta_{13}$ and one in the determination of the electron (or up quark) mass in SU(5) models. We have also considered the case in which correlations are present in the charged lepton mass matrix and, in the context of the possibility discussed in [14], we have identified the additional conditions that allow to solve the $m_{e}$ tuning problem in $\mathrm{SU}(5)$ models.
[1] R. Barbieri, T. Hambye, and A. Romanino, J. High Energy Phys. 03, 017 (2003).

[2] E.K. Akhmedov, G.C. Branco, and M.N. Rebelo, Phys. Rev. Lett. 84, 3535 (2000).

[3] S.M. Barr and I. Dorsner, Nucl. Phys. B585, 79 (2000).

[4] F. Feruglio, A. Strumia, and F. Vissani, Nucl. Phys. B637, 345 (2002).

[5] S. Lavignac, I. Masina, and C.A. Savoy, Nucl. Phys. B633,
139 (2002)

[6] P.H. Frampton, S.T. Petcov, and W. Rodejohann, Nucl. Phys. B687, 31 (2004).

[7] R. Kuchimanchi and R.N. Mohapatra, Phys. Lett. B 552, 198 (2003).

[8] R. Barbieri, P. Creminelli, and A. Romanino, Nucl. Phys. B559, 17 (1999).

[9] A. Ibarra and G.G. Ross, Phys. Lett. B 575, 279 (2003). 
[10] R.F. Lebed and D.R. Martin, hep-ph/0312219.

[11] S.F. King and N.N. Singh, Nucl. Phys. B596, 81 (2001).

[12] S.F. King, J. High Energy Phys. 09, 011 (2002).

[13] The present paper is based on a talk first given at CERN in September 2003.

[14] G. Altarelli, F. Feruglio, and I. Masina, Nucl. Phys. B689, 157 (2004).

[15] A.Y. Smirnov, Phys. Rev. D 48, 3264 (1993); S.F. King, Phys. Lett. B 439, 350 (1998); S. Davidson and S.F. King, ibid. 445, 191 (1998); R. Barbieri, L.J. Hall, and A. Strumia, ibid. 445, 407 (1999); Q. Shafi and Z. Tavartkiladze, ibid. 451, 129 (1999); S.F. King, Nucl. Phys. B562, 57 (1999); G. Altarelli, F.
Feruglio, and I. Masina, Phys. Lett. B 472, 382 (2000); S.F. King, Nucl. Phys. B576, 85 (2000).

[16] C.H. Albright and S.M. Barr, Phys. Rev. D 58, 013002 (1998); C.H. Albright, K.S. Babu, and S.M. Barr, Phys. Rev. Lett. 81, 1167 (1998); G. Altarelli and F. Feruglio, Phys. Lett. B 439, 112 (1998); P.H. Frampton and A. Rasin, ibid. 478, 424 (2000).

[17] S.F. King, Rep. Prog. Phys. 67, 107 (2004).

[18] R. Gatto, G. Sartori, and M. Tonin, Phys. Lett. 28B, 128 (1968); R.J. Oakes, ibid. 29B, 683 (1969); 30B, 262 (1969).

[19] R. Barbieri, L.J. Hall, S. Raby, and A. Romanino, Nucl. Phys. B493, 3 (1997). 